\title{
Fatores críticos de sucesso na implantação da governança da tecnologia da informação na administração pública federal
}

\author{
André Luiz Nascimento Reis \\ Universidade Católica de Brasília (UCB) \\ João Souza Neto \\ Universidade Católica de Brasília (UCB/DF)
}

A pesquisa teve como objetivo identificar fatores críticos de sucesso (FCS) na implantação da governança da tecnologia da informação (GTI) na administração pública federal brasileira (APF). Foram extraídos da literatura fatores críticos na implantação da GTI. Por meio do método Delphi, especialistas indicaram o grau de criticidade de cada fator. Foram considerados FCS aqueles que obtiveram consenso e apresentaram menor variação dos resultados. Foram identificados dez FCS, são eles: a alta administração deve apoiar continuamente a implantação; a implantação da GTI deve ser decidida pela alta administração; alinhar a TI aos negócios; deve haver comunicação e cooperação eficiente entre as partes interessadas; identificar e envolver partes interessadas chave; iniciar com alguns processos; a gestão de recursos de TI deve ter dimensão estratégica na organização; reter pessoas chave para a implantação; capacitar a equipe de TI para adotar mudanças; e, monitorar conformidades legais. Os dez FCS foram apresentados, por meio de grupo focal, a gestores de organizações pertencentes à APF para que opinassem sobre a pertinência dos mesmos nesse contexto. Como resultado, constatou-se que os FCS relacionados à alta administração foram considerados os mais relevantes, e que os FCS identificados mostraramse aderentes ao contexto da APF. federal

Palavras-chave: governança de TI, fatores críticos de sucesso, administração pública

[Artigo recebido em 28 de setembro de 2018. Aprovado em 15 de outubro de 2019.] 
Factores de éxito críticos en la implementación de la gobernanza de la tecnología de la información en la administración pública federal

El objetivo de la investigación fue identificar los factores de éxito críticos (FEC) en la implementación de la gobernanza de la TI (GTI), en la administración pública federal de Brasil (APF). Los factores críticos en la implantación de la GTI se extrajeron de la literatura. Usando el método Delphi, se les pidió a los expertos que indicaran el grado de criticidad de cada factor. Los FEC considerados fueron los que obtuvieron consenso y presentaron una variación menor de los resultados. Se han identificado diez FCS, que son: la alta gerencia debe apoyar continuamente la implementación; la implementación de la ITN debe ser decidida por la alta gerencia; alinear TI con el negocio; debe haber una comunicación y cooperación eficiente entre las partes interesadas; identificar e involucrar a las partes interesadas clave; comience con algunos procesos; la gestión de recursos de TI debe tener una dimensión estratégica en la organización; retener a las personas clave para el despliegue; empoderar al personal de TI para aceptar el cambio; y, supervisar el cumplimiento legal. Los diez FEC identificados fueron presentados a través de un grupo focal a los gerentes de las organizaciones que pertenecen a la APF para expresar su opinión sobre su relevancia en este contexto. Como resultado, se descubrió que los FEC relacionados con la alta administración se consideraron los más relevantes y los FEC identificados adhirieron al contexto de la APF.

Palabras clave: gobernanza de $\mathrm{TI}$, factor de éxito crítico, administración pública federal

\section{Critical success factors in implementing information technology governance in federal public administration}

The aim of the research was to identify the critical success factors (CSF) in the implementation of IT governance (ITG), in the Brazilian federal public administration (FPA). The critical factors in the implantation of the GTI were extracted from the literature. Using the Delphi method, experts were asked to indicate the degree of criticality of each factor. The CSF considered were those that obtained consensus and presented smaller variation of the results. Ten CSF have been identified, which are: top management should continually support deployment; the implementation of the ITG must be decided by senior management; align IT with business; there must be efficient communication and cooperation between stakeholders; identify and engage key stakeholders; start with some processes; IT resource management must have a strategic dimension in the organization; retain key people for deployment; empower IT staff to embrace change; and, monitor legal compliance. The ten FCS were presented, through a focus group, to managers of organizations belonging to the FPA to give their opinion on their relevance in this context. As a result, it was found that senior management-related CSF were considered to be the most relevant and that identified CSF were adherent to the FPA context.

Keywords: IT Governance, critical success factor, federal public administration 


\section{Introdução}

Na última década, a governança tem atraído a atenção das organizações devido à sua importância no provimento de respostas aos desafios dos negócios globais e aos interesses de suas diversas partes interessadas, tornando-se uma preocupação predominante para executivos, acadêmicos e políticos em todo o mundo (CLAESSENS; YURTOGLU, 2013).

Governança é um sistema de direcionamento e controle, sendo a governança corporativa (GC) compreendida como um leque de políticas relacionadas às funções de direção e controle das corporações (INTERNATIONAL ORGANIZATION FOR STANDARDIZATION, 2015), de forma a criar e prover informações transparentes e de alta qualidade (CLAESSENS; YURTOGLU, 2013).

Nos últimos anos, as organizações têm aumentado sua dependência de tecnologia da informação (TI), principalmente porque a maioria das operações é efetuada em ambientes informatizados (MENDONÇA et al., 2013). A TI tornou-se parte integral de todas as funções de negócio, sendo tão significativa quanto qualquer outra área e, em muitas organizações, tornou-se crucial no apoio, sustentabilidade e crescimento dos negócios (De Haes; Van Grembergen, 2009; Pereira; Ferreira, 2015).

Portanto, administrar com eficiência os recursos de TI tornou-se um desafio (BERMEJo et al., 2014) a ser superado com o propósito de agregar valor ao negócio, atingir os objetivos estratégicos e obter benefícios para a organização (DE FARIA et al., 2015). Assim, busca-se assegurar que a TI entregue benefícios dentro dos prazos propostos e dos custos e investimentos estabelecidos (TEODoro et al., 2014).

Por meio da implantação de uma bem concebida infraestrutura de governança de tecnologia da informação (GTI), a TI busca a eficiência na gestão de seus recursos (DE FARIA et al., 2015). Entende-se, também, a GTI como um subconjunto da GC, pelo qual o uso atual e futuro da TI é dirigido e controlado (JACOBSON, 2009). Assim, a GTI tornou-se fator essencial para a gestão financeira e estratégica de uma organização e não mero suporte (SORTICA et al., 2004).

$\mathrm{Na}$ administração pública federal brasileira (APF) a TI alcançou status de destaque. Prova disso é que o Tribunal de Contas da União (TCU), desde 2007, realiza levantamentos com o objetivo de realizar um diagnóstico sobre a situação da GTI na APF, com foco na verificação da conformidade e do desempenho, por meio de análises sistemáticas de informações relativas a governança, segurança e aquisições de bens e de serviços de TI (BRASIL, 2015).

Os levantamentos realizados pelo TCU apontam para uma tendência de evolução nas iniciativas de implantação de GTI na APF. Contudo, o próprio TCU argumenta que essa situação está distante do desejado (BRASIL, 2015). Apesar de uma evolução, 
a situação da GTI na APF permanece não desejada, e está longe de ser aceitável (BRASIL, 2016, 2017, 2018). Esse cenário gera questionamentos sobre como vem sendo feita a implantação da GTI na APF, o que sugere lacunas nas orientações para essa implantação.

A falta de orientações objetivas para a implantação da GTI na APF, tendo em vista as particularidades e especificidades do setor público (poder e legitimidade), (KLUMB; AZEVEDo, 2014), apresenta-se como problema a ser enfrentado. Principalmente por que os principais esforços são despendidos na etapa de implementação (ALREEMY et al., 2016).

Essa falta de orientações impacta as organizações públicas, impedindo a apropriação de lições aprendidas e comprometendo a efetividade das implantações. Adicionalmente à falta de orientações mencionada, Rodrigues e Souza Neto (2012) argumentam que há poucas referências sobre GTI no setor público, indicando que esse tema deve ser melhor explorado.

Considerando fatores críticos de sucesso (FCS) como elementos essenciais para a implementação efetiva da GTI, verifica-se que poucos estudos sobre FCS foram realizados e que pesquisas nessa área podem ser usadas para melhorar as abordagens disponíveis para implementação de governança de TI no setor público (NFUKA; RUSU, 2011).

Diante do exposto, emergiu a seguinte questão: quais são os FCS que devem ser considerados na implantação da GTI na APF? Para responder a essa questão foi realizada uma pesquisa que identificou os fatores críticos presentes na implantação da GTI, de forma geral. Os FCS identificados foram submetidos, por meio do método Delphi, a análises de especialistas, que atribuíram um grau de criticidade a eles e indicaram quais FCS estão presentes na APF. Posteriormente, especialistas analisaram, por meio de grupo focal, como os FCS ocorreram no contexto da APF. Assim, a pesquisa teve como objetivo a identificação de FCS na implementação da GTI na APF, por meio da confirmação empírica de diferentes elementos presentes na literatura.

O artigo possui cinco seções, conforme segue: introdução, referencial teórico (onde foram abordados os temas governança corporativa, GTI, GTI na APF e FCS), método, análise e discussões dos resultados, e conclusão.

\section{Referencial teórico}

\subsection{Governança corporativa}

A GC vem se estabelecendo como um campo de pesquisa desde os anos 70 (FISS, 2008). Em sua origem, a GC está ligada à necessidade de representatividade 
por parte de pequenos acionistas de empresas que eram impotentes, uma vez que apenas alguns investidores institucionais acumulavam uma quantidade de ações que lhes permitiam ter espaço nas decisões corporativas (MORCK; STEIER, 2005).

A GC pode ser definida como um conjunto de mecanismos que regem o processo decisório em uma organização para garantir o retorno adequado a seus investidores e evitar que haja algum tipo de ação por parte de seus agentes contrária a esse objetivo, sendo esses agentes indivíduos ou outras organizações contratados pelos proprietários ou controladores para gerir uma organização (CARVALHO, 2002).

O conceito de GC pode ser ampliado acrescentando temas como processos, costumes, políticas, leis e até instituições como potenciais influenciadores das decisões em uma organização (LUNA et al., 2013). Tal ampliação do conceito também é observada no código do Instituto Brasileiro de Governança Corporativa, que acrescenta outras partes interessadas, indo muito além dos interesses dos acionistas (INSTITUTO BRASILEIRO DE GOVERNANÇA CORPORATIVA, 2015).

No Brasil, há um movimento para que os padrões de GC sejam aprimorados, destacando-se que tais padrões têm aplicação tanto em organizações públicas quanto em organizações privadas, guardadas as suas peculiaridades (INSTITUTO BRASILEIRO DE GOVERNANÇA CoRPorATIVA, 2015). Particularmente, em relação às organizações públicas, espera-se, primordialmente, o cumprimento de orçamentos e prazos (OMARI et al., 2012).

Depreende-se, assim, que o conceito de GC abrange um conjunto de processos que permitem dirigir, monitorar e incentivar o relacionamento entre as diversas partes interessadas internas e externas de uma organização, de forma a se alcançarem os objetivos desejados por cada uma dessas partes.

\subsection{Governança da tecnologia da informação}

Em função das características do setor público, uma maior ênfase foi dada à responsabilização e ao controle, passando a ser esse o foco da GTI (TEODORO et al., 2014). Tal situação se reflete nos conceitos e definições encontrados na literatura, onde constata-se que, em praticamente todas as definições, há expressões (e derivadas ou similares) como "controlar" e "monitorar" que remetem à ideia de garantia de atingimento dos objetivos organizacionais, como pode ser visto no Quadro 1. 


\section{Quadro 1 - Definições de GTI}

$\begin{array}{ll}\text { Autor(es) } & \text { Definição } \\ \text { (PETERSON, 2004) } & \text { Sistema de gerenciamento corporativo por meio do qual } \\ \text { o portfólio de TI de uma organização é direcionado e } \\ \text { controlado. } \\ \text { Parte integral da governança corporativa que provê } \\ \text { mecanismos para a liderança, estruturas e processos } \\ \text { (NFUKA; RUSU, 2010) } \\ \text { organizacionais que garantam que a TI da organização } \\ \text { sustente as estratégias e objetivos organizacionais. } \\ \text { Uma visão de governança que garante que a informação e } \\ \text { a tecnologia relacionada apoiem e possibilitem a estratégia } \\ \text { da organização e a consecução dos objetivos corporativos. } \\ \text { Também inclui a governança funcional de TI, ou seja, a que } \\ \text { garante que as capacidades de TI sejam fornecidas com } \\ \text { eficiência e eficácia. }\end{array}$

(BERMEJo et al., 2014)

GTI como a especificação da tomada de decisão, estruturas, processos e mecanismos relacionais de orientação e controle.

Sistema de distribuição de responsabilidades e direitos sobre

(TEODORO et al., 2014) decisões de $\mathrm{TI}$, e de gerenciamento e controle dos recursos tecnológicos da organização, buscando garantir o alinhamento da $\mathrm{TI}$ às estratégias e aos objetivos organizacionais.

Compreende o conjunto de mecanismos para assegurar que

(BRASIL, 2015) o uso da TI agregue valor ao negócio das organizações, com riscos aceitáveis.

(ISO/IEC, 2015)

Sistema pelo qual o uso atual e futuro da TI é dirigido e controlado.

A GTI vai além das responsabilidades específicas das (PEREIRA; Ferreira, tecnologias de informação, expandindo-se aos aspetos de 2015) negócio relacionados à $\mathrm{TI}$, necessários à criação de valor para a organização.

A governança de $\mathrm{Tl}$ é o processo que orienta e controla os

(ALREEMY et al., 2016) investimentos, decisões e práticas relacionadas à TI dentro da organização, a fim de alcançar os objetivos desejados.

Fonte: elaboração própria.

A TI vem se desenvolvendo de uma maneira muito rápida, a ponto de penetrar em toda a organização, transformando os processos de negócios internos e externos, possibilitando o atendimento das expectativas dos clientes e a permanência no mercado (BHATT; EMdAD, 2010; MANFREdA; SteMBERger, 2014). Tal realidade sugere que o foco em controle e monitoramento dado pela GTI, herdado da GC, é apropriado, o que indica que a implantação de um adequado sistema de GTI por parte das organizações é um movimento de importância estratégica. 
Contudo, a implantação de um sistema de GTI requer um esforço de integração da organização, sendo necessária uma série de interações para que possa ser implantada adequadamente e gerar valor para a organização (ALREEMY et al., 2016). Dessa forma, o princípio básico da GTI é o alinhamento entre TI e negócio, sendo esse alinhamento utilizado como ferramenta para mitigar as frustrações decorrentes do mau uso dos investimentos em TI e obter vantagens competitivas (RAMOS, 2015).

\subsection{Governança de tecnologia da informação na administração pública federal brasileira}

Além da necessidade de legitimação das decisões, há de se considerar que a GTI em organizações do setor público visa à geração de benefícios para a população e outras partes interessadas (SANTOS; SOUZA NETO, 2014). Essas organizações têm objetivos por vezes intangíveis, como saúde, segurança e educação, que devem ser entregues e/ou disponibilizados considerando restrições de prazo e orçamento (OMARI et al., 2012).

O principal impulsionador da adoção de práticas de GTI na APF é o TCU, órgão responsável por auxiliar o Congresso Nacional no exercício do controle externo (BRASIL, 2012). Reforçando o fato de que a GTI faz parte da boa governança das empresas públicas (JUIz et al., 2014),, o TCU realiza levantamentos sobre a adoção de práticas de GTI, por meio de questionários enviados às organizações.

O primeiro levantamento foi realizado em 2007, quando foi identificado um cenário de preocupação. No segundo levantamento, em 2010, foi criado o índice de governança de $\mathrm{TI}$ (iGovTI) para orientar as organizações na melhoria de suas práticas de GTI, permitindo a avaliação da efetividade das práticas adotadas. Em 2012, quando foi realizado o terceiro levantamento, constatou-se que houve uma evolução no cenário da GTI, na APF, contudo, foi verificada a adoção insuficiente de práticas fundamentais de GTI (BRASIL, 2015), sinalizando uma situação ainda distante do ideal. Em 2016, novamente o TCU apontou uma evolução, mas, ressaltou que a situação da GTI na APF está distante de patamares aceitáveis (BRASIL, 2016).

Em 2017, o TCU passou a acompanhar a GTI de forma integrada com outras governanças (pessoas, contratações e resultados), uma vez que, para o TCU, a governança e a gestão perpassam todas as funções organizacionais. Como os resultados do iGovTI de 2016 foram disponibilizados somente em meados de 2017, o TCU optou por não calcular o iGovTI 2017, mas, mantendo a avaliação da GTI. Em 2018 o iGovTI voltou a ser calculado. Contudo, tanto em 2017 quanto em 2018, a GTI na APF perdura em uma situação distante do aceitável (BRASIL, 2017), apresentando, de forma geral, um cenário não desejado, considerando a sua importância para o desempenho das organizações (BRASIL, 2018). 
Conforme Ramos (2015), os levantamentos realizados pelo TCU são considerados como o principal instrumento de avaliação da GTI na APF. A partir desses levantamentos é que são elaborados os acórdãos do TCU com determinações visando melhorar a GTI na APF, todavia, esses levantamentos não orientam sobre a implantação da GTI, nem apontam os FCS a serem considerados nessa implantação.

\subsection{Fatores críticos de sucesso}

Segundo Rockart (1979), o Dr. Ronald Daniel introduziu, em 1961, o conceito de FCS, citando que grandes corporações produzem um grande número de informações e que apenas um pequeno grupo de informações auxiliavam efetivamente os gestores na melhoria do desempenho. Para que fosse dada atenção a esse pequeno grupo de informações, foi introduzido o conceito de FCS. Foi verificado por Caralli (2004) que o conceito de FCS também pode ser útil em aplicações diversas e não somente no campo de sistemas de informações, sendo necessária sua adaptação para cada tipo de indústria/segmento.

FCS são aplicados a um número limitado de áreas em que os resultados devem ser satisfatórios para garantir o desempenho esperado de uma organização. Ou seja, se os resultados nessas áreas não forem adequados, a organização não alcançará seus objetivos (ROCKART, 1979). O conceito de FCS apresentado por Caralli (2004) amplia essas possibilidades, postulando que esses são mais do que áreas, perpassando atividades, rotinas e elementos culturais.

Há também fatores que, embora sejam considerados importantes, não são críticos. Isso acontece quando o cumprimento de determinado objetivo é alcançado com a ocorrência ou não desses fatores, embora sua presença aumente a probabilidade de sucesso. São fatores considerados desejáveis e a eles é dada a denominação de fatores de sucesso (FS) (CUNHA; SOUZA NETO, 2014).

Para esta pesquisa, o conceito de FCS utilizado foi o de que eles são elementos chave de desempenho para que uma organização alcance seus objetivos e cumpra sua missão. Destaca-se que a má execução desses elementos faz com que a organização não atinja seus objetivos e, consequentemente, não cumpra sua missão (ALREEMY et al., 2016). FCS podem ser práticas, estratégias, táticas, métodos, ferramentas, técnicas, elementos culturais e motivacionais que precisam ser bem executados para que uma organização tenha aumentadas as suas probabilidades de sucesso.

Os FCS podem ser classificados em três dimensões distintas (BULLEN; ROCKART, 1981). Na primeira dimensão, a origem, os FCS podem surgir de cinco fontes primárias: a) indústria - cada tipo de indústria, segmento ou setor possui seus FCS, aos quais as organizações devem se atentar; b) estratégica competitiva dependendo de sua estratégia ou de sua posição no mercado, a organização pode 
ter diferentes FCS; c) ambiente competitivo - são FCS sobre os quais a organização não possui influência direta e que estão ligados ao ambiente competitivo no qual a organização está inserida; d) temporalidade - há situações em que os FCS emergem circunstancialmente, por um período de tempo, devido a situações diversas; e) gestores funcionais - cada gestor funcional possui o seu conjunto de FCS.

Quanto à segunda dimensão, os FCS podem ser classificados como internos ou externos, sendo que a principal característica dos fatores internos é que eles estão relacionados a situações onde os gestores possuem influência/controle. Já a principal característica dos fatores externos é que eles estão relacionados a situações onde há pouca ou nenhuma influência/controle por parte dos gestores.

A terceira dimensão classifica os FCS de acordo com sua finalidade, podendo ser classificados como de: a) monitoramento - fatores que demandam monitoramento constante para acompanhar o desempenho organizacional; b) construção/adaptação - fatores que estão relacionados à adaptação da organização ao enfrentamento de novas situações e/ou ambientes.

Além de suas dimensões, os FCS também possuem uma hierarquia que retrata as relações entre cada um desses fatores (GATES, 2010). Os níveis hierárquicos entre os FCS são: indústria; organizacional; departamental; operacional e individual. Os FCS de indústria influenciam as organizações de uma determinada indústria (segmento ou setor) no desenvolvimento da sua estratégia, seus objetivos e suas metas. Essas organizações desenvolvem estratégias que contemplam os seus principais FCS organizacionais. Estes últimos, por sua vez, direcionam os gestores para a identificação de FCS departamentais, que direcionam os FCS operacionais, sendo esses dois últimos suportados pelos FCS individuais.

Como método, os FCS obtiveram aprovação dos executivos de mercado na medida em que definem processos que demandam menor tempo e apresentam melhores resultados (ROCKART, 1979). Dada sua perspectiva de aplicação em várias áreas de conhecimento e sua possibilidade de aplicações diversas, há várias técnicas e métodos que podem ser utilizados para a identificação de FCS. Destacase o questionário do tipo survey como o método mais comumente utilizado para identificar FCS (ESTEVES DE SOUZA, 2004).

Como benefícios, o método FCS permite que o gestor determine os fatores que deverão demandar maior atenção e esforço, desenvolva medidas adequadas para esses fatores, defina claramente a quantidade de recursos que devem ser alocados pela organização, acomode mudanças na estratégia em função de alterações ambientais e as aplique em várias áreas afetas ao processo de gestão (ROCKART, 1979). 


\section{Metodologia}

A presente pesquisa pode ser classificada como quantitativa e qualitativa de caráter descritivo, uma vez que buscou identificar e entender o significado e a ocorrência dos FCS na implantação da GTI na APF, dado que há poucas referências sobre GTI no setor público (Peterson, 2002; Rodrigues; Souza Neto, 2012), sugerindo que esse tema seja melhor explorado (CRESWELL, 2010).

A execução da pesquisa se deu em quatro fases. A primeira fase foi a identificação de FCS nas implantações de GTI presentes na literatura, aplicados em diversos contextos. A segunda fase foi a realização de uma avaliação, por meio do método Delphi, junto a especialistas em GTI na APF, para que os fatores identificados na literatura fossem analisados e classificados sob o ponto de vista de sua criticidade no contexto da APF.

O método Delphi é uma ferramenta para obter julgamentos coletivos sobre determinado assunto junto a especialistas independentes (HUANG et al., 2013). É um método flexível, interativo e de facilitação de grupo realizado em vários estágios (rodadas) de modo a trocar opinião individual por consenso em grupo, (HASSON et al., 2000). Em resumo, o método Delphi consiste na realização de rodadas anônimas de questionários postais ou por e-mail (caso do presente estudo), coleta de feedback individual e resumo dos resultados, repetindo esse processo até que seja alcançado determinado nível de consenso ou até que seja realizado um número predeterminado de rodadas (HUMPHREY-MURTo e colab., 2017). No método Delphi os participantes não interagem diretamente preservando o anonimato de cada participante, de modo que não haja influências e constrangimentos (MCMILLAN et al., 2016).

$\mathrm{Na}$ terceira fase, foi realizado um grupo focal com gestores de TI da APF, com o objetivo de verificar as percepções sobre a pertinência dos FCS identificados e contextualizá-los na realidade de organizações vinculadas à APF (GOMES; BARBOSA, 1999). Por fim, na quarta fase, os FCS foram classificados segundo as dimensões e hierarquias propostas por Bullen e Rockart (1981) e Gates (2010). Na pesquisa, foi utilizado o método de abordagem de FCS disseminado por Rockart (1979), uma vez que mostrou-se viável, dada a sua versatilidade para aplicação em áreas distintas.

\subsection{Coleta de dados}

A coleta de dados ocorreu no período compreendido entre março e agosto de 2016. A identificação de FCS na implantação da GTI, presentes na literatura, buscou por práticas ou processos ou FCS na implantação da GTI em qualquer tipo de organização. Os FCS identificados na literatura foram submetidos a vinte 
especialistas em GTI, por meio do método Delphi, para que fossem analisados e classificados sob o ponto de vista de sua criticidade (baixíssima, baixa, média, alta e altíssima) (CUNHA; SouzA Neto, 2014). Para isso, foram enviados questionários via e-mail, utilizando o software Survey Monkey. A escolha dos especialistas se deu por meio de indicação.

Além da classificação quanto à criticidade, os especialistas foram estimulados a realizar observações e indicar eventuais FCS não identificados. Constavam também nos questionários questões relativas ao tempo de experiência (em anos) na área de TI de órgão/empresa pública, de modo a confirmar se os especialistas possuíam o perfil desejado. Por fim, foi solicitado que informassem sua ocupação para caracterização do perfil. Dois especialistas em TI que não participaram da pesquisa realizaram a validação dos questionários em cada uma das rodadas.

O método Delphi foi finalizado após a realização de duas rodadas. Os especialistas que responderam ao questionário da primeira rodada foram convidados a participar da segunda rodada. Cada especialista recebeu um relatório de feedback, contendo um resumo dos resultados da primeira rodada e a compilação das respostas de todos os especialistas em comparação com as suas respostas, além de gráficos para uma melhor visualização das informações.

Após a identificação dos FCS, foi realizado um grupo focal com cinco gestores de $\mathrm{TI}$, com, em média, mais de 17 anos de experiência, que atuam em organizações pertencentes à APF, principalmente do setor judiciário. Os especialistas que participaram do método Delphi não foram convidados a participar do grupo focal, para que não houvesse algum tipo de viés em defesa de opiniões já emitidas.

\subsection{Tratamento dos dados}

Para a identificação dos FCS na literatura, foi adotada, como critério de seleção, a abordagem de FCS na implantação da GTI ou de FCS na implantação de alguma prática (framework) (Information Technology Infrastructure Library - ITIL, por exemplo), desde que fosse citado explicitamente pelo(s) autor(es) que a prática foi utilizada para implantação de GTI, uma vez que a utilização de uma ou combinações de várias práticas pode ser utilizada para a implantação da GTI (ALREEMY et al., 2016; RODRÍGUEZ; VIEIRA, 2007). Os FCS identificados foram analisados conforme suas descrições, significados e agrupados em categorias (ALREEMY et al., 2016), de forma a possibilitar um melhor entendimento. FCS com descrições e significados semelhantes foram compilados e tiveram suas descrições unificadas.

Para cômputo dos resultados do método Delphi, foi atribuído um valor para cada nível de criticidade atribuído por cada especialista, para cada FCS, sendo: 1 (baixíssima), 2 (baixa), 3 (média), 4 (alta) e 5 (altíssima). As respostas dos 
especialistas para cada fator foram divididas em três grupos, conforme a frequência de opiniões sobre o fator: a) favoráveis - somatório das opiniões com criticidade alta + altíssima; b) indiferentes - somatório das opiniões com criticidade média e d) desfavoráveis - somatório das opiniões com criticidade baixíssima + baixa. Nas duas rodadas, o consenso foi considerado quando o somatório das opiniões dos especialistas atingisse $75 \%$ em um dos grupos citados.

A classificação geral dos fatores considerou os seguintes critérios: a) fator crítico de sucesso - percentual das opiniões "favoráveis" maior ou igual a 90\% e coeficiente de variação (variação dos valores em torno da média) maior ou igual a 0,15; b) fator de sucesso - percentual das opiniões "favoráveis" maior ou igual a $90 \%$ e coeficiente de variação maior ou igual a 0,3; c) não FCS - percentual de opiniões "favoráveis" menor que 90\% (CUNHA; SOUZA NETO, 2014).

Os participantes do grupo focal receberam um código para identificação de suas falas. Um facilitador conduziu a apresentação dos tópicos na reunião e três auxiliares o apoiaram no direcionamento das discussões. As falas dos participantes foram gravadas e analisadas manualmente por meio de análise conteúdo (BARDIN, 2011). As percepções dos participantes foram agrupadas conforme categorias dos FCS e reproduzidas em afirmações que refletiam a convergência de opiniões do grupo para cada um dos FCS.

\section{Resultados e discussões}

O perfil dos especialistas consultados foi predominantemente (80\%) de servidores públicos ou empregados de empresas estatais com, no mínimo, 10 e, em média, 24 anos de experiência em GTI, perfil esse considerado adequado aos objetivos da pesquisa. Utilizou-se o coeficiente alpha de Cronbach para análise da consistência interna dos questionários respondidos pelos especialistas, sendo respectivamente 0,9009 para a primeira rodada e 0,8157 para a segunda, estando ambos acima do valor mínimo sugerido de 0,7 (HAIR et al., 2009).

Foram identificados quarenta e quatro fatores, que foram submetidos à análise dos especialistas na primeira rodada do método Delphi. Ao final da primeira rodada, vinte e cinco fatores identificados apresentaram consenso, oito foram classificados como FCS e dezenove não apresentaram consenso, sendo necessária uma segunda rodada. Ainda na primeira rodada, foram apresentadas, pelos especialistas, observações acerca dos fatores.

Os fatores que não apresentaram consenso foram submetidos a uma segunda rodada, quando, também, foram enviadas as observações feitas pelos especialistas. Ao final da segunda rodada, foram identificados dez FCS, outros dez foram 
classificados como FS, e vinte e quatro foram classificados como não FCS. Os dez FCS identificados após a segunda rodada do método Delphi estão apresentados no Quadro 2, por ordem de criticidade, e classificados em categorias, seguidos dos autores.

Os dois fatores que compõem o grupo alta administração (F1 - A alta administração deve apoiar continuamente a implantação e F2 - A implantação da GTI deve ser decidida pela alta administração) foram classificados como FCS, ressaltando a relevância da participação da alta administração na implantação da GTI em organizações da APF. Tal relevância também foi verificada nos relatos do grupo focal e está aderente aos achados da literatura, que consideraram como FCS a participação da alta administração na implantação da GTI em um contexto mais amplo (AHMAD et al., 2013; HeIER et al., 2008; IDEN; EIKEBROKK, 2014; MEHRAVANI et al., 2011; NICHO; MOURAD, 2012; WANG; SERESHKI, 2010) e no contexto do setor público (NFUKA; RUSU, 2010).

O fator F3 - deve haver comunicação e cooperação eficiente entre as partes interessadas, mostrou-se de grande influência na implantação da GTI na APF uma vez que, segundo as discussões do grupo focal, é importante que todos entendam o papel da GTI e que os gestores entendam os benefícios da implantação, corroborando com os achados de Nfuka e Rusu (2010).

\section{Quadro 2 - FCS na implantação da GTI na APF}

\begin{tabular}{|c|c|c|}
\hline Fator e descrição & Categorias & Autor(es) \\
\hline $\begin{array}{l}\text { A alta administração deve } \\
\text { apoiar continuamente a } \\
\text { implantação }\end{array}$ & $\begin{array}{l}\text { Alta } \\
\text { administração }\end{array}$ & $\begin{array}{l}\text { (AHMAD e colab., 2013; HEIER } \\
\text { et al., 2008; IDEN; EIKEBROKK, } \\
\text { 2014; MEHRAVANI et al., 2011; } \\
\text { NFUKA; RUSU, 2010; NICHO; } \\
\text { MOURAD, 2012) }\end{array}$ \\
\hline $\begin{array}{l}\text { A implantação da GTI } \\
\text { deve ser decidida pela alta } \\
\text { administração }\end{array}$ & $\begin{array}{l}\text { Alta } \\
\text { administração }\end{array}$ & $\begin{array}{l}\text { (IDEN; EIKEBROKK, 2014; WANG; } \\
\text { SERESHKI, 2010) }\end{array}$ \\
\hline $\begin{array}{l}\text { Deve haver comunicação e } \\
\text { cooperação eficiente entre as } \\
\text { partes interessadas }\end{array}$ & Comunicação & $\begin{array}{l}\text { (AHMAD et al., 2013; HEIER } \\
\text { et al., 2008; IDEN; EIKEBROKK, } \\
\text { 2014; NFUKA; RUSU, 2010; } \\
\text { NICHO; MOURAD, 2012; WANG; } \\
\text { SERESHKI, 2010) }\end{array}$ \\
\hline Alinhar a TI aos negócios & Estratégia & (SHIVASHANKARAPPA et al., 2012) \\
\hline $\begin{array}{l}\text { Identificar e envolver partes } \\
\text { interessadas chave }\end{array}$ & $\begin{array}{l}\text { Partes } \\
\text { interessadas }\end{array}$ & $\begin{array}{l}\text { (IDEN; EIKEBROKK, 2014; } \\
\text { NICHO; MOURAD, 2012; WANG; } \\
\text { SERESHKI, 2010) }\end{array}$ \\
\hline
\end{tabular}




\begin{tabular}{|c|c|c|}
\hline $\begin{array}{l}\text { Reter pessoas chave para a } \\
\text { implantação }\end{array}$ & $\begin{array}{l}\text { Partes } \\
\text { interessadas }\end{array}$ & (SHIVASHANKARAPPA et al., 2012) \\
\hline Iniciar com alguns processos & Processos & $\begin{array}{l}\text { (IDEN; EIKEBROKK, 2014; WANG; } \\
\text { SERESHKI, 2010) }\end{array}$ \\
\hline $\begin{array}{l}\text { Monitorar conformidades } \\
\text { legais }\end{array}$ & Requisitos & (SHIVASHANKARAPPA et al., 2012) \\
\hline $\begin{array}{l}\text { Capacitar a equipe de TI para } \\
\text { adotar mudanças }\end{array}$ & Treinamento & $\begin{array}{l}\text { (HEIER et al., 2008; IDEN; } \\
\text { EIKEBROKK, 2014; NFUKA; RUSU, } \\
\text { 2010) }\end{array}$ \\
\hline $\begin{array}{l}\text { A gestão de recursos de TI } \\
\text { deve ter dimensão estratégica } \\
\text { na organização }\end{array}$ & Estratégia & (NFUKA; RUSU, 2010) \\
\hline
\end{tabular}

Fonte: elaboração própria.

O fator F4, alinhamento da Tl aos negócios, apresentou aderência ao alinhamento da GTI como governança funcional da GC, sugerindo que não basta uma GTI eficiente, é preciso que ela esteja alinhada ao planejamento estratégico global da organização. O princípio básico da GTI é o alinhamento entre TI e negócio, sendo esse alinhamento utilizado como ferramenta para mitigar as frustrações decorrentes do mau uso dos investimentos em TI e para obter vantagens competitivas (RAMOS, 2015). Tal achado também reforça o papel da GTI como subconjunto da GC, apoiando o cumprimento da missão organizacional (JACOBSON, 2009; LUNA et al., 2013).

A identificação e o envolvimento das partes interessadas chave (F5) foram classificados como FCS pelos especialistas, ratificando as considerações de Iden e Eikebrokk (2014), Nicho e Mourad (2012) e Wang e Sereshki (2010), ficando patente, no grupo focal, que a comunicação exerce um importante papel na implantação da governança, chegando ao ponto de, por falta de comunicação, as pessoas não saberem qual é o objetivo da GTI.

Durante a realização do grupo focal um ponto levantado nas discussões foi a existência de "campeões" para a implantação da GTI, o que, na literatura, foi denominado como "definir um campeão". Esse fator não foi classificado como FCS. Contudo, esses "campeões" foram citados no grupo focal como pessoas chave e a retenção dessas pessoas se confirmou como FCS (F6 - reter pessoas chave para a implantação) (SHIVASHANKARAPPA et al., 2012).

O fator F7 - iniciar com alguns processos, foi tratado no grupo focal como diretamente relacionado com a escassez de recursos. Dado que não há recursos para iniciar ao mesmo tempo todos os processos planejados, esses processos 
devem ser priorizados e implantados paulatinamente, tal qual proposto por Iden e Eikebrokk (2014) e Wang e Sereshki (2010).

A capacitação da equipe de TI, F9 - capacitar a equipe de TI para adotar mudanças (AHMAD e colab., 2013; IDEN; EIKEBROKK, 2014), também foi ratificada no grupo focal como FCS necessário à implantação da GTI na APF. Adicionalmente, foi ressaltado pelos participantes do grupo focal que o esforço empreendido para capacitar a equipe de TI deve ser acompanhado paralelamente por um esforço de capacitação da alta administração, como forma de aproximação da alta administração com os técnicos.

A gestão de recursos de TI com dimensão estratégica na organização ( $F$ 10) está diretamente associada à atuação da alta administração no provimento dos recursos necessários à implantação da GTI na APF e tal atuação foi reforçada nos relatos do grupo focal, corroborando Nfuka e Rusu (2010), que afirmaram que a alta administração deve atuar de forma a alocar os recursos escassos eficazmente para melhorar os processos de TI.

Diversos autores relacionam FCS à adoção e utilização de framework (principalmente Itil, Cobit e ISO, dentre outros) para a implantação da GTI (ALREEMY et al., 2016; IDEN; EIKEBRoKK, 2014; Kumsuprom, 2010; MeHRAVANI et al., 2011; Nicho; Mourad, 2012; ShIVASHANKARAPPA et al., 2012; WANG; SERESHKI, 2010). A frequência dessa relação fez com que um fator denominado "Um framework de GTI deve ser adotado" fosse adotado na pesquisa. Apesar disso, esse fator não foi considerado um FCS nem, tampouco, foi citado no grupo focal.

Nos estudos realizados por Heier, Borgman e Hofbauer (2008) é apresentado como problema a dificuldade das empresas na implantação de software de GTI, uma vez que muitas das iniciativas não cumprem os prazos nem os custos estabelecidos. Fatores relacionados ao cumprimento de prazos e custos não foram classificados como FCS nem citados no grupo focal.

Para De Haes e Van Grembergen (2006), a diferença entre o ambiente público e o privado impacta a GTI, uma vez que organizações do setor privado são tipicamente mais flexíveis em termos de orçamento, decisões de pessoal e procedimentos, enquanto as organizações públicas são caracterizadas por procedimentos rígidos, dependência de apontamentos políticos etc. Questões relacionadas a esses itens não foram verificadas durante a realização do grupo focal, sugerindo que a rigidez nos procedimentos na APF é considerada como norma a ser cumprida, não cabendo discussão quanto à sua adoção.

Além da identificação dos FCS e da discussão de sua pertinência no grupo focal, eles foram classificados de acordo com as dimensões propostas por Bullen e Rockart (1981) e estão apresentados na Figura 1, onde cada FCS está identificado por 
seu número, conforme numeração do Quadro 2. Tal classificação e apresentação mostraram-se pertinentes, uma vez que não foram identificados na literatura estudos que tivessem essa abordagem, limitando-se a identificar os FCS.

\section{Figura 1 - FCS identificados e suas dimensões}

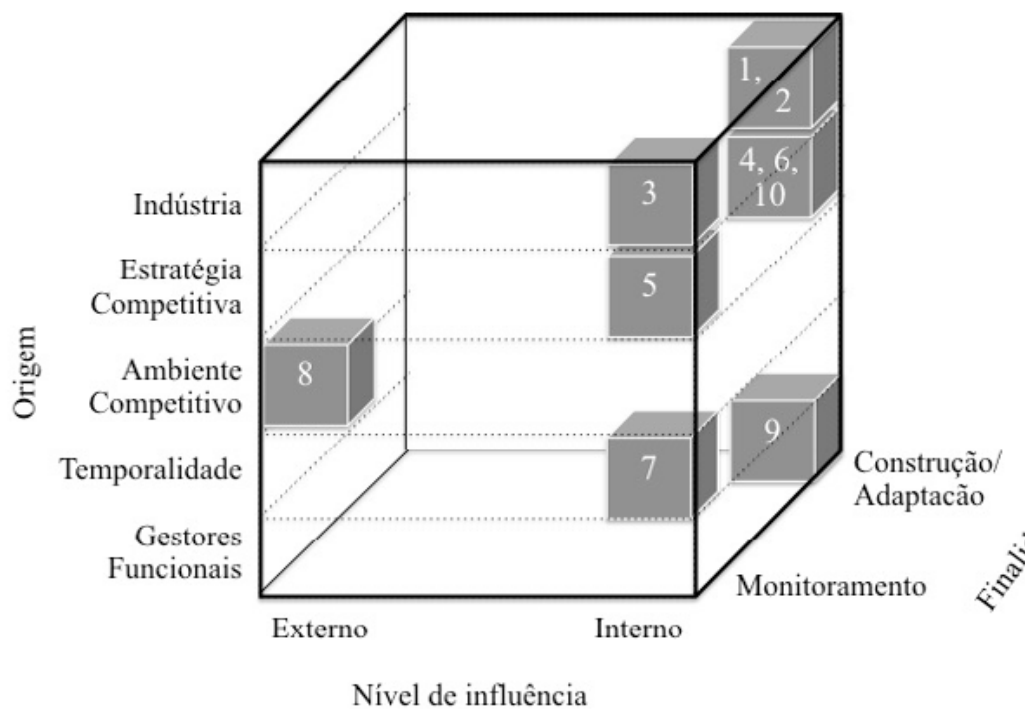

Fonte: elaboração própria.

Considerando a classificação dos FCS constantes da Figura 1, verifica-se que houve um equilíbrio entre fatores classificados como de monitoramento e de construção/adaptação, sugerindo que as organizações devem manter suas atividades operacionais e de rotina sob controle, ao mesmo tempo que devem se preparar para os desafios futuros.

O foco da $\mathrm{TI}$ na responsabilização e no controle, conforme apresentado por Teodoro, Przeybilovicz e Cunha (2014) ficou evidenciado nos resultados da pesquisa, uma vez que o fator F8 - monitorar conformidades legais, foi classificado como FCS. Esse foco foi ratificado nos relatos do grupo focal, tendo o TCU um papel importante nessas adoções, o que corroborou o posicionamento de Ramos (2015) de que aquele órgão, por meio de suas avaliações de gestão, desempenha o principal papel na avaliação da GTI na APF. Esse cenário fica evidenciado na Figura 1, quando somente o fator F8 apresenta dimensão externa.

Em relação à hierarquia proposta por Bullen e Rockart (1981) e Gates (2010), nota-se que há prevalência dos FCS organizacionais, seguidos pelos de indústria, e que não há FCS classificados como individuais, conforme o Quadro 3. Tal fato pode ser explicado pela exigência de cumprimento de normas e ritos burocráticos 
- legitimidade das decisões (KLUMB; AZEVEDo, 2014) - ser mais acentuado na APF, em detrimento de anseios individuais.

\section{Quadro 3 - Hierarquia dos FCS na implantação da GTI na APF}

\begin{tabular}{ll} 
Hierarquia & Fator e descrição \\
& F1-A alta administração deve apoiar continuamente a implantação \\
Indústria & F2 - A implantação da GTI deve ser decidida pela alta administração \\
& F4 - Alinhar a TI aos negócios \\
& F3 - Deve haver comunicação e cooperação eficiente entre as \\
& partes interessadas \\
& F5 - Identificar e envolver partes interessadas chave \\
& F7 - Iniciar com alguns processos \\
Organizacional & F10 - A gestão de recursos de TI deve ter dimensão estratégica na \\
& organização \\
& F6 - Reter pessoas chave para a implantação \\
& F9 - Capacitar a equipe de TI para adotar mudanças \\
\hline Departamental & F8 - Monitorar conformidades legais \\
\hline Operacional & -
\end{tabular}

Os fatores com origem na própria indústria (APF) e na estratégia competitiva prevaleceram sobre os demais. Essa constatação alinhada à hierarquia dos fatores apresentada na Quadro 3, onde prevalecem os FCS de indústria e organizacionais, sugere que a implantação da GTI na APF deve possuir caráter estratégico, conforme proposto por Nfuka e Rusu (2010).

\section{Conclusão}

O objetivo da pesquisa foi a identificação de FCS na implementação da GTI na APF, por meio da confirmação empírica de diferentes elementos presentes na literatura. Para isso, foram identificados na literatura os FCS na implantação da GTI. Esses FCS foram agrupados e especialistas opinaram quanto ao seu nível de criticidade. Adicionalmente, foram colhidas percepções de gestores de organizações pertencentes à APF sobre os FCS identificados no contexto da implantação da GTI. Por fim, um rol de dez FCS a serem considerados quando da implantação da GTI na 
APF foi proposto. Foi verificado que os fatores identificados relacionam-se entre si e não devem ser analisados de forma isolada.

A maioria (nove dos dez) dos FCS foram classificados como internos às organizações. Quanto à sua finalidade, eles estão divididos em FCS ligados à manutenção das atividades de rotina e ligados à preparação das organizações para desafios futuros. Não foram encontrados na literatura estudos que realizassem classificações similares para que fosse possível realizar análises comparativas. Essa lacuna pode ser explorada em estudos futuros.

Como limitação, tem-se que os FCS abordados possuem caráter temporal, sendo referências para o cenário atual da GTI na APF. Além disso, os FCS também variam conforme o grau de maturidade de cada organização. Sob o ponto de vista metodológico, ressalta-se que a realização de outros grupos focais, com a participação de mais gestores representantes dos Poderes Executivo e Legislativo, enriqueceria mais o trabalho.

Em trabalhos futuros, sugere-se a realização de estudos que verifiquem os relacionamentos e influências que um FCS possui em outro e em que grau, bem como o comportamento da evolução dos FCS ao longo do tempo, conforme a evolução do grau de maturidade das organizações pertencentes à APF. Ainda considerando que os FCS relacionam-se entre si, sugere-se a utilização de outras metodologias para sua identificação, por exemplo, a utilização de recursos de análise fatorial.

Sob o ponto de vista acadêmico, o estudo buscou preencher a lacuna existente entre os referenciais teóricos e a pesquisa empírica de forma que os resultados motivem futuros esforços de pesquisa sobre os mecanismos de GTI no contexto da APF. Sob o ponto de vista social, espera-se que esse rol de FCS possam encorajar gestores de organizações da APF, em particular do setor público em geral, a rever a eficácia de seus processos de GTI, bem como auxiliar aqueles que pretendem iniciar a implantação da GTI em busca de melhores resultados organizacionais, contribuindo em última instância para o aumento da eficiência na aplicação dos recursos públicos.

Por fim, conforme sugerem os resultados, pode-se concluir que os FCS mostraramse aderentes ao contexto das organizações ligadas à APF brasileira, sendo aplicáveis a esse contexto. 
Referências bibliográficas

AHMAD, Norita et al. Technology adoption model and a road map to successful implementation of ITIL. Journal of Enterprise Information Management, v. 26, p. 553-576, 2013.

ALREEMY, Zyad et al. Critical success factors (CSFs) for information technology governance (ITG). International Journal of Information Management, v. 36, n. 6, p. 907-916, 2016.

BARDIN, Laurence. Análise de Conteúdo. 1 de 2011 ed. São Paulo: Edições 70, 2011. BERMEJO, Paulo Henrique de Souza et al. Evaluating IT governance practices and business and IT outcomes: a quantitative exploratory study in Brazilian companies. Procedia Technology, B1-C, v. 16, p. 849-857, 2014.

BHATT, Ganesh D; EMDAD, Ali F. An empirical examination of the relationship between information technology (IT) infrastructure, customer focus, and business advantages. Journal of Systems and Information Technology, v. 12, n. 1, p. 4-16, 2.fev. 2010.

BRASIL. Análise da capacidade de governança e gestão em 526 órgãos e entidades da administração pública federal. Brasília, Brasil: Tribunal de Contas da União, 2018.

Constituição da República Federativa do Brasil de 1988. Brasília, Brasil: Câmara dos Deputados, 2012.

Levantamento de governança de TI 2014. Brasília: TCU, Secretaria de Fiscalização de Tecnologia da Informação, 2015.

. Levantamento em 581 órgãos e entidades da administração pública federal sobre governança e gestão em 2017. Brasília, Brasil: Tribunal de Contas da União, 2017.

Relatório de levantamento. Brasília, Brasil: TCU, Secretaria de Fiscalização de Tecnologia da Informação, 2016.

BULLEN, Christine V. ; ROCKART, John F. A primer on critical success factors. Working papers, n. 69, p. 1-64, 1981.

CARALLI, Richard A. The critical success factor method: establishing a foundation for enterprise security management. Working papers, n. , p. 1-118, july.2004.

CARVAlHo, Antônio Gledson De. Governança corporativa no Brasil em perspectiva. Revista de Administração, v. 37, n. 3, p. 19-32, 2002.

CLAESSENS, Stijn; YURTOGLU, B. Burcin. Análise da capacidade de governança e gestão em 526 órgãos e entidades da administração pública federal. Emerging Markets Review, v. 15, p. 1-33, 2013.

CResWell, J. W. Projeto de pesquisa: métodos qualitativo, quantitativo e misto. 3. ed. Porto Alegre: Artmed, 2010.

Cunha, Marcelo Silva; Souza Neto, João. Comitês de governança de tecnologia da informação na administração pública federal brasileira: fatores críticos de sucesso. Revista do Serviço Público, v. 65, n. 3, p. 355-381, 2014. 
DE FARIA, Maximiliano M. et al. Analyzing IT governance initiatives with game theory: a systematic literature review. Journal of Software, v. 10, n. 9, p. 1056-1069, 2015.

De HAES, Steven; VAN GREMBERGEN, Wim. Information technology governance best practices in Belgian organizations. 2006: IEEE, p. 195b,2006.

De HaEs, Steven; VAN Grembergen, Win. Exploring the relationship between IT governance practices and business/IT alignment through extreme case analysis in Belgian mid-to-large size financial enterprises. Journal of Enterprise Information Management, v. 22, n. 5, p. 615-637, 2009.

ESTEVES DE SOUZA, José Manuel. Definition and analysis of critical success factors for ERP implementation projects. 2004. 1-313 f. 2004. Tese de Doutorado. Universitat Politècnica de Catalunya, Barcelona, Spain., 2004.

FISS, Peer C. Institutions and corporate governance. The sage handbook of organizational institutionalism, p. 389-410, 2008.

GATES, Linda Parker. Strategic planning with critical success factors and future scenarios: an integrated strategic planning framework. Software Engineering Institute, n. november, p. 67, 2010.

GOMES, Maria Elasir S; BARBOSA, Eduardo F. A técnica de grupos focais para obtenção de dados qualitativos. Instituto de Pesquisas e Inovações Educacionais, p. 1-7, 1999. HAIR, J. F. et al. Análise multivariada de dados. Porto Alegre: Bookman, 2009.

HASSON, Felicity; KEENEY, Sinead ; MCKENNA, Hugh. Research guidelines for the Delphi survey technique. Journal of advanced Nursing, v. 32, n. 4, p. 1008-1015, 2000.

Heier, Hauke; Borgman, Hans P. ; HofBauer, Thomas H. Making the most of IT governance software: understanding implementation processes. In: 2008, Waikoloa, Estados Unidos: IEEE, 2008. p. 1-11.

HUANG, Sun-Jen; WU, Ming-Shian; CHEN, Li-Wei. Critical success factors in aligning IT and business objectives: a Delphi study. Total Quality Management and Business Excellence, v. 24, n. 10, p. 1219-1240, 2013.

HUMPHREY-MURTO, Susan et al. Using consensus group methods such as Delphi and nominal group in medical education research. Medical Teacher, v. 39, n. 1, p. 14-19, 2017.

Instituto Brasileiro de Gestão Corporativa (IBGC). Código das Melhores Práticas de Governança Corporativa. 5. ed. São Paulo: IBGC, 2015.

IDEN, Jon; EIKEBROKK, Tom Roar. Using the ITIL process reference model for realizing IT governance: an empirical investigation. Information Systems Management, v. 31, n. 1, p. 37-58, 2014.

ISACA. COBIT 5 - Modelo corporativo para governança e gestão de TI da organização. São Paulo, Brasil: São Paulo Chapter with the permission of ISACA, 2012.

ISO/IEC. ISO/IEC 38500:2015 Information technology - governance of IT for the organization. International Organization for Standardization, 2015. 
JACOBSON, Dax D. Revisiting IT governance in the light of institutional theory. Proceedings of the 42nd Annual Hawaii International Conference on System Sciences, HICSS, p. 1-9, 2009.

JUIZ, Carlos; GuerRero, Carlos ; LeRA, Isaac. Implementing good governance principles for the public sector in information technology governance fameworks. Open Journal of Accounting, v. 3, n. jan., p. 9-27, 2014.

KLUMB, Rosangela; AZEVEDo, Beatriz Marcondes De. A percepção dos gestores operacionais sobre os impactos gerados nos processos de trabalho após a implementação das melhores práticas de governança de TI no TRE/SC. Revista de Administração Pública, v. 48, n. 4, p. 961-982, 2014.

KUMSUPROM, Siridech. Structured approach to organisational ICT risk management : an empirical study in thai businesses. 2010. 353 f. RMIT University, 2010.

LUNA, Alexandre J.H. de O.; KRuChten, Philippe; De MourA, Hermano P. Game: governance for agile management of enterprises: a management model for agile governance. Ago. 2013: IEEE, p. 88-90,ago.2013.

MAnfredA, Anton; Stemberger, Mojca Indihar. Factors causing the relationship gap between top management and IS personnel. Journal of Enterprise Information Management, v. 27, n. 2, p. 107-121, 4 fev. 2014.

MCMILLAN, Sara S. ; KING, Michelle; Tully, Mary P. How to use the nominal group and Delphi techniques. International Journal of Clinical Pharmacy, v. 38, n. 3, p. 655-662, 2016.

MehravanI, Sarvenaz; HaJIHeydarI, Nastaran; HaghIghINASAB, Manijeh. ITIL Adoption Model based on TAM. 2011, Singapore: [s.n.], p. 33-37, 2011.

MEndonçA, Cláudio Márcio Campos De et al. Governança de tecnologia da informação: um estudo do processo decisório em organizações públicas e privadas. Revista de Administração Pública, v. 47, n. 2, p. 443-468, 2013.

MORCK, Randall K; STEIER, Lloyd. The global history of corporate governance : an introduction. 2005, Chicago: University of Chicago Press, p. 65,2005.

NFUKA, E. N. ; RUSU, Lazar. Critical success factors for effective IT governance in the public sector organizations in a developing country: the case of Tanzania, 2010.

NFUKA, E N ; RUSU, Lazar. The effect of critical success factors on IT governance performance. Industrial Management \& Data Systems, v. 111, n. 9, p. 1418-1448, 2011.

NICHO, Mathew ; MouRAD, Basel Al. Success factors for integrated ITIL deployment: an IT governance classification. Journal of Information Technology Case and Application Research, v. 14, n. 1, p. 25-54, 2012.

OMARI, Loai Al; BARNes, Paul H; PITMAN, Grant. Optimising COBIT 5 for IT governance : examples from the public sector. 2012, Taipei, Taiwan: academy of Taiwan information systems research, p. 1-13,2012.

PEREIRA, Cristiano ; FERREIRA, Carlos. Identificação de práticas e recursos de gestão do valor das TI no COBIT 5. RISTI - Revista Ibérica de Sistemas e Tecnologias de Informação, n. 15, p. 17-33, jun. 2015. 
Peterson, Ryan. Crafting information technology governance. Information Systems Management, v. 21, n. 4, p. 7-22, 2004.

PETERSON, Ryan. Information technology governance processes under environmental dynamism: investigating competing theories of decision making and knowledge sharing. IE Working Paper. Madrid, Spain, 2002.

RAMOS, Karoll Haussler Carneiro. Análise multivariada de fatores críticos de sucesso em governança de Ti na administração pública federal à luz dos dados de controle externo. 2015. 229 f. Universidade de Brasília, Brasil, 2015.

ROCKART, John F. Chief executives define their own data needs. Harvard business review, v. 57, n. 2, p. 81-92, 1979.

RODRIGUES, José Geraldo Loureiro; SOUZA NETO, João. Diretrizes para implantação da governança de Ti no setor público brasileiro à luz da teoria institucional. Revista do Serviço Público, v. 63, n. 4, p. 475-497, 2012.

Rodríguez, Martius Vicente Rodriguez y; VIEIRA, Daniele Machado. Governança de TI no setor publico - Caso da Dataprev. Revista Produção Online, v. 7, n. 1, p. 207225, 2007.

SANTOS, Diana Leite Nunes Dos; SouzA Neto, João. Avaliaçãoda capacidade dos processos de governança corporativa de TI baseada no COBIT 5. Revista Eletrônica de Sistemas de Informação, v. 13, n. 01, p. 18, 30 abr. 2014.

SHIVASHANKARAPPA, Arun Nagarle et al. Implementing it governance using cobit: a case study focusing on critical success factors. 2012, Guelph, Canadá: IEEE, . 144149,2012.

Sortica, E. ; Clementi, S; CARvalho, T. Governança de TI: comparativo entre COBIT e ITIL. 2004.

Teodoro, Allexandre Nishioka; Przeybilovicz, Erico; CunhA, Maria Alexandra. Governança de tecnologia da informação: uma investigação sobre a representação do conceito. Revista de Administração, v. 49, n. 2, p. 307-321, 2014.

WANG, Jingwen; SERESHKI, Hami Khosravi. How to implement ITIL successfully? 2010. 77 f. Jönköping University, Suécia, 2010.

André Luiz Nascimento Reis

Universidade Católica de Brasília (UCB). Doutorando em Administração pela UnB. Mestre em Gestão do Conhecimento e da Tecnologia da Informação pela Universidade Católica de Brasília. Pós-Graduado em Strategic Management of Competitiveness and Innovation for the Executive Manager pela Universidade de Miami. MBA em Gestão de Projetos pela Universidade de Vila Velha/ES. Bacharel em Administração de Empresas, com habilitação em Análise de Sistemas, pela Faculdade de Ciências Humanas de Vitória/ES. Bacharel em Administração Postal pela Escola Superior de Administração Postal. uai@hotmail.com; http://lattes.cnpq.br/3748387386409400; https:// orcid.org/0000-0002-7389-7643

\section{João Souza Neto}

Universidade Católica de Brasília (UCB/DF). Doutor em Engenharia Elétrica pela Universidade de Brasília. Mestre em Engenharia Eletrônica pelo Philips International Institute da Holanda. Professor do Mestrado em Governança, Tecnologia e Inovação da Universidade Católica de Brasília. sznetoj@gmail.com.; http://lattes.cnpq. $\underline{\mathrm{br} / 7010050556592203}$ 